\title{
Preditores da qualidade de vida em fumadores e abstinentes
}

\author{
Natália Andrade \\ Maria da Graça Pereira \\ Universidade do Minho - Braga, Portugal
}

\begin{abstract}
Resumo
Este estudo teve como objetivo avaliar qualidade de vida, morbilidade psicológica, dependência nicotínica e coping familiar em fumadores e abstinentes. 116 fumadores e 117 abstinentes, de ambos os sexos, responderam individualmente aos instrumentos: Sociodemográfico; Medical Outcomes Study Short-Form 36 (MOS SF-36); Escala de Ansiedade Depressão e Stress (EADS); Fagerström Test for Nicotine Dependence (FTND) e Family Crises-Oriented Personal Evaluation Scales (FCOPES). Os resultados revelaram que ter parceiro fumador, elevada dependência nicotínica e morbilidade psicológica predizem menor qualidade de vida física. Elevada morbilidade psicológica e avaliação passiva são preditores de pior qualidade de vida mental. Quanto aos abstinentes, doença respiratória crónica, morbilidade psicológica e procura de apoio espiritual predizem menor qualidade de vida física. Ser do sexo feminino, possuir doença respiratória crónica e morbilidade psicológica predizem menor qualidade de vida mental. Futuramente, programas de intervenção devem incluir parceiros e incidir na morbilidade psicológica e coping para aumentar a qualidade de vida.
\end{abstract}

Palavras-chave: Fumadores, Abstinentes, Qualidade de vida, Morbilidade psicológica.

\section{Life quality predictors in smokers and abstinents}

\begin{abstract}
This study aimed to evaluate quality of life, psychological morbidity, nicotine dependence and family coping in smokers and abstinents. 116 smokers and 117 abstinents, from both sexes, participated in the study. All participants answered the following instruments: Socio-Demographic; Medical Outcomes Study Short Form 36 (MOS SF-36); Depresion, Anxiety, Stress Scales (DASS); Fagerström Test for Nicotine Dependence (FTND) and Family Crises-Oriented Personal Evaluation Scales (F-COPES). According to the results, in smokers who also have a partner who smokes, high nicotine dependence and psychological morbidity predict less physical quality of life. High psychological morbidity and passive assessment, predict less quality of life. As for abstinents, having chronic respiratory disease, high psychological morbidity and looking for spiritual support predicted poorer quality of physical life. Being female, having chronic respiratory disease and psychological morbidity predicted lower mental quality of life. Hereafter, intervention programs should include partners as well as psychological morbidity and coping strategies in order to increase quality of life.
\end{abstract}

Keywords: Smokers, Abstinents, Quality of life, Psychological morbidity.

\section{Predictores de la calidad de vida en fumadores y abstinentes}

\section{Resumen}

La presente investigación tuvo como objetivo evaluar la calidad de vida, morbilidad psicológica, dependencia nicotínica y ayuda familiar en fumadores y abstinentes. 116 fumadores y 117 abstinentes, de ambos los sexos, respondieron de forma individual a los cuestionarios: Socio demográfico; Medical Outcomes Study Short-Form 36 (MOS SF-36); Escala de Ansiedad, Depresión y Stress

Endereço para correspondência: Escola de Psicologia (EPSI), Campus de Gualtar, 4710-057. Braga, Portugal. Email: nat_sofia@msn.com. Telefone/Fax: +351 253604220.

Artigo aceito durante a gestão do editor Gerson Tomanari. 
(EADS); Fagerström Test for Nicotine Dependence (FTND) y Family Crises-Oriented Personal Evaluation Scales (F-COPES). Los resultados revelaron que tener conyugue fumador, elevada dependencia nicotínica y morbilidad psicológica predicen menor calidad de vida física. Elevada morbilidad psicológica y evaluación pasiva predicen una peor calidad de vida mental. En los abstinentes, la enfermedad respiratoria crónica, morbilidad psicológica y la busca de apoyo espiritual predicen menor calidad de vida física. Ser del sexo femenino, tener alguna enfermedad respiratoria crónica y morbilidad psicológica predicen menor calidad de vida mental. En el futuro, los programas de intervención deben incluir a los conyugues e incidir en la morbilidad psicológica y en las estrategias de enfrentamiento para aumentar la calidad de vida.

Palabras clave: Fumadores, Abstinentes, Calidad de vida, Morbilidad psicológica.

\section{Introdução}

O tabagismo difere dos restantes consumos de substâncias psicoativas pela ausência, a curto prazo, de efeitos mortais, de patologia mental ou de comportamento desadaptado, o que leva à desvalorização da real gravidade problemática associada ao comportamento tabágico percebido pela grande maioria dos fumadores como normal e inofensivo (Martinet \& Bohadana, 2003). Segundo a Direção Geral de Saúde (DGS) (2009), entre os anos 2005 e 2006, cerca de $19.6 \%$ da população portuguesa era fumadora, dos quais $28.7 \%$ eram homens e $11.2 \%$ eram mulheres, havendo maior incidência entre as pessoas com idade entre os 35 e os 44 anos. Por sua vez, os abstinentes representavam cerca de $15.4 \%$ da população portuguesa, sendo que $24.9 \%$ eram do sexo masculino e $6.6 \%$ do sexo feminino. Entre as pessoas que deixaram de fumar, verificou-se maior prevalência de abstinentes com idade entre os 55 e os 64 anos. Fazendo um paralelismo com os dados de 1999, verifica-se uma diminuição da percentagem de fumadores masculinos e um aumento da prevalência de fumadoras. Por ano, registramse cerca de quatro milhões de mortes associadas ao consumo do tabaco, a nível mundial, sendo a principal causa de mortes evitáveis (WHO, 2002).

O principal benefício da desabituação tabágica consiste na redução da morbilidade do indivíduo, melhorando assim a sua qualidade de vida (Sarna, Bialous, Cooley, Jun, \& Feskanich, 2008). Em contexto de saúde e doença, a qualidade de vida engloba uma análise subjetiva do bem-estar físico, mental e social que pode variar no tempo (WHO, 2002). A longa duração de consumo de tabaco e o número elevado de cigarros fumados por dia associam-se à reduzida qualidade de vida
(Efstratiadis, Kennard, Kelsey, \& Michaels, 2008; Sarna et al., 2008) e quanto maior a dependência, mais forte tende a ser esta relação (Schmitz, Kruse, \& Kugler, 2003). Após 10 anos de consumo, surgem sintomas subtis e, após 20 a 30 anos, são visíveis problemas físicos graves (Machain et al., 2008). O tabagismo é associado a numerosas doenças crónicas como úlceras, cancros, doenças cardiovasculares e respiratórias (Doherty, Grabowski, Hoffman \& Zelikoff, 2009). Os fumadores apresentam também maior risco de desenvolverem diabetes tipo II e, por sua vez, complicações da diabetes, como a resistência à insulina e a síndrome metabólica, podem ser exacerbadas pelo tabagismo (Tonstad, 2009).

Machain et al. (2008) defendem que, à medida que o hábito tabágico se prolonga no tempo e os problemas de saúde física são agravados, aumentam os níveis de distress emocional dos fumadores. Segundo Dube et al. (2009), abstinentes apresentam níveis de distress psicológico mais reduzidos que fumadores. Wagena et al. (2004) verificaram que os resultados mantiveram-se perante $o$ controlo da existência de diagnóstico de doença crónica, necessário, devido ao fato de os indivíduos com patologia crónica apresentarem frequentemente perturbações psicológicas. Quanto à recaída, a razão principal é a dificuldade em lidar com o stress (Siahpush, Spittal, \& Singh, 2007). A prevalência de distress psicológico tende a ser mais elevada nos adultos fumadores, com baixo nível socioeconómico (NSE) e entre 45 a 64 anos de idade (Pratt, Day, \& Cohen, 2007). Por outro lado, adultos com elevado distress psicológico tendem a fumar mais (Hagman, Delnevo, Hrywna \& Williams, 2007) e elevados níveis de ansiedade têm sido associados a uma intensidade mais elevada da percepção de sintomas de abstinência, dificultando o 
processo de cessação tabágica (Gonzalez, Zvolensky, Vujanovic, Leyro, \& Marshall, 2009). Assim, o uso da nicotina tem vindo a ser relacionado com redução da ansiedade, stress e severidade da depressão (Machain et al., 2008). Porém, fumar também agrava o afeto negativo quando o tabagismo ocorre na presença de humor deprimido. A reduzida tolerância ao distress reforça o comportamento tabágico e a história de depressão reforça o tabagismo independentemente do estado de humor (Perkins, Karelitz, Giedgowd, Conklin, \& Sayette, 2010). Vários estudos revelam que indivíduos com história de perturbações de humor, ansiedade ou de abuso de substâncias têm mais tendência a fumar, relatar sintomas de abstinência e recaídas do que indivíduos sem perturbação (Weinberger, Desai \& Mckee, 2010). Apesar de muitos dos fumadores relatarem que usam o tabaco como meio de reduzir a ansiedade, a investigação tem revelado que o uso crônico da nicotina relaciona-se positivamente com elevados níveis de ansiedade (Feldner et al., 2009). Fumadores com elevado distress psicológico são mais dependentes da nicotina (Hagman et al., 2007) e têm mais dificuldades na cessação tabágica, o que leva ao aumento de distress psicológico e depressão major (Machain et al., 2008).

A literatura define o tabagismo como uma toxicodependência classificada como perturbação psicológica, cujas causas podem ser sociais, psicológicas, comportamentais e farmacológicas (Ezquerra, 2003), manifestando-se como uma doença crónica (WHO, 2002). À medida que o consumo de nicotina aumenta, surge a dependência física (Foulds \& Ghodse, 1995). A nicotina atinge o cérebro após 10 segundos da inalação, difundese no cérebro e ativa os receptores nicotínicos. Ocorre a libertação de dopamina no núcleos accumbens e nas áreas límbica e estriatal associadas a sentimentos de prazer e ao aumento da atividade motora (Wonnacott, Drasdo, Sanderson, \& Rowell, 1990), bem como a libertação de norepinefrina (aumento da ativação), serotonina (regulação do humor), GABA (redução da ansiedade e da tensão músculo-esquelética), acetilcolina (aumento dos estados de alerta, concentração e função cognitiva) e glutamato (aumento da retenção da memória e da capacidade de aprendizagem) (Benowitz, 1999, 2008). Se a nicotina deixa de ser consumida, o indivíduo experiencia a síndrome de abstinência (Bergen \& Caporaso,
1999). Como já foi referido, os efeitos da nicotina podem alterar as emoções, o comportamento ou as cognições. Estes efeitos parecem estar na base da dependência psicológica. Mas, de acordo com os índices fisiológicos, a nicotina aumenta a estimulação em todas as circunstâncias. O que varia é a percepção subjetiva do indivíduo influenciada pelo seu contexto ambiental (Perkins et al., 2010), ou seja, na dependência da nicotina, estão envolvidos fatores psicológicos e sociais que influenciam o modo como os indivíduos percepcionam os efeitos físicos (Martinet \& Bohadana, 2003). Assim, os reforços subjetivos da nicotina são associados a situações, emoções e contextos. Fumar é um comportamento repetitivo regulado por recompensas imediatas através do reforço positivo (obtenção de prazer) ou negativo (evitar sintomas da abstinência) (Ezquerra, 2003). A dificuldade do desmame está positivamente relacionada com o nível de dificuldades económicas, pessoais e sociais (Graham, 1993). A investigação realça que a iniciação, manutenção e cessação do comportamento tabágico são fortemente influenciadas pela família (McDaniel, Campbell, Hepworth \& Lorenz, 2005).

Visto que o tabagismo pode ser considerado uma doença crónica (WHO, 2002) a noção de coping familiar torna-se pertinente. Segundo McCubbin, Patterson e Sussman (1983), o coping familiar consiste numa resposta dos membros da família aos stressores, que abrange interações e movimentações dos membros da família para gerir dificuldades, resolver conflitos e tensões intrafamiliares, bem como adquirir e desenvolver recursos sociais, psicológicos e materiais, necessários à adaptação familiar.

A adaptação da família nuclear à doença relaciona-se com a procura de apoio social e o uso de uma comunicação aberta entre os seus membros (Kershaw, Northouse, Kritpracha, Shafenacker, \& Mood, 2004). Segundo Holm, LaChance, Bowler, Make e Wamboldt (2010), o apoio social dos familiares é importante para o sucesso na cessação tabágica, sendo fundamental o apoio do parceiro e familiares mais próximos. Os comportamentos negativos do companheiro predizem a recaída, visto que os relacionamentos familiares influenciam o surgimento e manutenção da ansiedade e da depressão e, por seu turno, os sintomas de depressão e ansiedade predizem o surgimento e manutenção do tabagismo (Patton et al., 1998), 
assim como aumentam o risco de recaída (Anda et al., 1990). A investigação realça que o comportamento tabágico entre parceiros tende a ser semelhante. A ocorrência de cessação tabágica de fumadores que convivem com fumadores e cujo parceiro seja fumador é menos provável (Rüge et al., 2008) e ter um companheiro não fumador ou abstinente relaciona-se com uma probabilidade maior de sucesso na cessação tabágica (Monden, de Graaf, \& Kraaykamp, 2003). Assim, a intenção para deixar de fumar relaciona-se com o tipo de comportamento tabágico do parceiro (Rüge et al., 2008).

Tendo em atenção a literatura existente, o nosso objetivo primordial foi conhecer as diferenças entre fumadores e abstinentes ao nível das dependência da nicotina, da morbilidade psicológica, da qualidade de vida e do coping familiar e quais os melhores preditores da qualidade de vida física e mental nos fumadores e abstinentes.

\section{Metodologia}

\section{Amostra}

O estudo incluiu 233 indivíduos dos quais 116 são fumadores com diagnóstico de Dependência de Nicotina segundo o DSM-IVTR e 117 são abstinentes há mais de 3 meses. A amostra foi recolhida numa universidade, num hospital e numa empresa privada do Norte de Portugal.

Entre os fumadores, $57.4 \%$ são homens e $42.6 \%$ são mulheres e no grupo dos abstinentes, $64.1 \%$ são homens e os restantes $35.9 \%$ são mulheres. A idade dos fumadores varia entre 18 e 54 anos (média de 31 anos de idade) e nos abstinentes, de 18 a 84 anos (média de 47 anos). $32.8 \%$ dos fumadores possuem 9 anos de escolaridade, $59.5 \%$ relatam ter 10 a 12 anos de escolaridade e $7.8 \%$ apresentam mais de 12 anos. Nos abstinentes, 59\% possuem 9 anos de escolaridade, $30.8 \%$ referem 10 a 12 anos de escolaridade e $10.3 \%$ têm mais de 12 anos. Quanto ao estado civil, $50.9 \%$ dos fumadores são solteiros, $31.6 \%$ são casados, $15.8 \%$ vivem em união de fato e $1.8 \%$ são divorciados. $75.2 \%$ dos abstinentes são casados, $4.3 \%$ vivem em união de fato, $14.5 \%$ são solteiros, $2.6 \%$ são viúvos e $3.4 \%$ são divorciados.

Neste estudo, $8.6 \%$ dos fumadores e $27.4 \%$ dos abstinentes relatam ter doença respiratória crónica. $37.1 \%$ dos fumadores nunca tentaram deixar de fumar e $51.3 \%$ dos abstinentes conseguiram a cessação tabágica na primeira tentativa. $47.4 \%$ dos fumadores relatam que o companheiro fuma e $52.6 \%$ referem que o parceiro não é fumador. Quanto aos abstinentes, $81.2 \%$ possuem um companheiro não fumador.

\section{Procedimento}

Após parecer favorável da Comissão de Ética da universidade, da empresa e do hospital, os sujeitos que desejaram participar no estudo foram contatados pelas investigadoras. Os participantes tiveram conhecimento do estudo através da informação deixada no local. Antes da aplicação individual dos instrumentos, todos os participantes assinaram o consentimento informado, no qual se garantiu o anonimato e a confidencialidade dos dados. Os fumadores que desejaram participar foram avaliados em termos de diagnóstico de dependência nicotínica por um psicólogo.

\section{Instrumentos}

- Questionário Sócio-Demográfico (Pereira \& Afonso, 2008b): Avalia idade, estado civil, sexo, anos de escolaridade e aspetos clínicos como: ter doença respiratória crónica, número de tentativas e hábito tabágico do companheiro de fumadores e abstinentes.

- Medical Outcomes Study Short Form 36 (MOS SF-36) (Ware \& Sherbourne, 1992 versão adaptada de Ferreira \& Santana, 2003): Constituído por 8 subescalas de resposta tipo Likert que avaliam Função Física (10 itens); Desempenho Físico (4 itens); Dor Corporal (2 itens); Saúde Geral (5 itens); Vitalidade (4 itens); Função Social (2 itens); Desempenho Emocional (3 itens) e Saúde Mental (5 itens). Estas dimensões agrupam-se em duas componentes gerais: Física (primeiras 4 dimensões) e Mental. Quanto mais elevada a pontuação, melhor a percepção de qualidade de vida. Não existe um valor total da escala, mas dois valores correspondentes às duas dimensões gerais supracitadas.

- Escala de Ansiedade, Depressão e Stress (EADS) (P. Lovibond \& S. Lovibond, 1995versão adaptada por Pais-Ribeiro, Honrado \& Leal, 2004): Tendo por base o Modelo Tripartido de Clark e Watson (1991), avalia as dimensões distress, ansiedade e depressão. Existem duas versões (42 e 21 itens). Neste estudo, foi usada a versão de 21 items agrupados em 3 subescalas. $\mathrm{Na}$ dimensão 
Depressão, enquadram-se os itens relativos à Disforia, Desânimo, Desvalorização da Vida, Autodepreciação, Falta de Interesse ou de Envolvimento, Anedónia e Inércia. Os itens referentes à Excitação do Sistema Autônomo, Efeitos Músculo-Esqueléticos, Ansiedade Situacional e Experiência Subjetiva de Ansiedade pertencem à Dimensão Ansiedade. E, finalmente, os itens representativos das variáveis Dificuldade em Relaxar, Excitação Nervosa, Impaciência, Facilmente Agitado/Chateado, Irritável/Reação Exagerada agrupam-se na dimensão Stress Psicológico. A cotação total varia de 0 a 21 e cada dimensão pode ser classificada como normal, leve, moderada, severa e muito severa. Pontuações mais elevadas correspondem a estados afetivos mais negativos (Pais-Ribeiro et al., 2004).

\section{Fagerström Test for Nicotine}

Dependence (FTND) (Fagerström, 1978; Fagerström \& Shneider, 1990 - versão de investigação de Pereira \& Afonso, 2008a): Instrumento de 6 itens. Nos itens 1 e 4 , a cotação varia de 0 a 3 e nos restantes de 0 a 1 , cujo somatório permite-nos classificar a dependência como leve ( 0 a 4$)$, moderada ( 5 a 7) e elevada (7 a 10), sendo que quanto mais elevada a pontuação, mais severa a dependência.

\section{Family Crises-Oriented Personal} Evaluation Scales (F-COPES) (McCubbin, Larsen \& Olson, 1982 - versão adaptada por Mendes et al., 1999): Avalia a percepção individual das atitudes e das estratégias de coping comportamental e de resolução dos problemas adotadas pelas famílias em resposta a situações problemáticas ou difíceis. A construção do F-COPES teve por base as dimensões de coping familiar abordadas no Modelo Duplo ABCX da teoria do stress familiar (McCubbin et al., 1983). O F-COPES avalia duas dimensões da interação familiar: o coping familiar interno e o coping familiar externo. Trata-se de uma escala de 30 itens que, após análises mais aprofundadas das suas características psicométricas, ficou reduzida a 29 itens com a eliminação do item 18. Os itens do instrumento são agrupados em 5 subescalas: Obtenção de Apoio Social (9 itens); Reenquadramento (8 itens); Procura de Apoio Espiritual (4 itens); Mobilização da Família para a Obtenção e Aceitação de Ajuda (4 itens) e Avaliação Passiva (4 itens). Quanto mais elevado o resultado, melhor coping familiar (Fisher \& Corcoran, 2007).

\section{Análise dos Dados}

$\mathrm{Na}$ análise das diferenças nas variáveis psicológicas, foram utilizados Testes- $t$ e Mancova (controlando a idade e os anos de escolaridade) quando os pressupostos da estatística paramétrica estavam cumpridos e, quando isso não aconteceu, testes não paramétricos de Man-Whitney e KruskalWallis.

No que concerne ao estudo dos preditores da qualidade de vida, foi realizada uma regressão Linerar (método Enter). No bloco I, foram inseridas as variáveis sociodemográficas e/ou clínicas e, no bloco II, foram acrescentadas as variáveis psicológicas, relacionadas com cada uma das variáveis dependentes.

\section{Resultados}

Diferenças na Dependência da Nicotina, Morbilidade Psicológica, Qualidade de Vida e Coping Familiar em função do Sexo, Idade, Anos de Escolaridade, Estado Civil, Presença de Doença Respiratória Crónica em Fumadores e Abstinentes.

Nos fumadores, verificam-se diferenças entre sexos ao nível da dependência da nicotina. Os homens revelam mais dependência tabágica. Ao nível do coping, na obtenção de apoio social existem também diferenças entre sexos $\left(\mathrm{F}_{(1.113)}=4.09, \mathrm{p} \leq .05\right)$, sendo as mulheres quem utiliza melhor a estratégia obtenção de apoio social.

Quanto aos abstinentes, observam-se diferenças significativas ao nível da dependência da nicotina, na medida em que são os homens que relatam níveis mais elevados de dependência da nicotina. No respeitante à qualidade de vida mental, existem diferenças significativas ao nível da vitalidade $\left(\mathrm{F}_{(1.111)}=4.38, \mathrm{p} \leq .05\right), \quad \mathrm{e}$ da saúde mental $\left(\mathrm{F}_{(1.111)}=4.28, \mathrm{p} \leq .05\right)$. $\mathrm{O}$ sexo masculino refere mais vitalidade e saúde mental.

Nos abstinentes, os resultados evidenciam diferenças significativas na dependência nicotínica, quando fumavam, em função da idade $\left(\chi_{(2)}^{2}=22.76, \mathrm{p} \leq .001\right)$. Os abstinentes com idade superior a 40 anos de idade, enquanto fumadores, percepcionam-se como mais dependentes da nicotina que os abstinentes com 40 ou menos anos.

Em relação aos anos de escolaridade, foram encontradas diferenças significativas ao nível da dependência da nicotina em função dos 
anos de escolaridade dos fumadores e dos abstinentes. Tanto fumadores, como abstinentes com pelo menos 9 anos de escolaridade relatam mais dependência da nicotina que aqueles que referem ter mais de 12 anos de escolaridade. Constatamos diferenças significativas nos fumadores ao nível da procura de apoio espiritual $\left(\chi^{2}=6.19, \quad \mathrm{p} \leq .05\right), \quad$ sendo que fumadores que possuam até 12 anos de escolaridade tendem a utilizar com mais frequência a estratégia de coping de procura de apoio espiritual. Nos abstinentes, há também diferenças ao nível da qualidade de vida física sendo superior em abstinentes com mais de 12 anos de escolaridade.

Quanto ao estado civil, ao nível das dimensões totais, não foram encontradas diferenças. Porém, nas subescalas, no grupos dos fumadores surgem diferenças, em termos de coping, ao nível da procura de apoio espiritual $\left(\mathrm{t}_{(112)=} 2.40, \mathrm{p} \leq .05\right)$ e, no grupo de abstinentes, são identificadas diferenças ao nível do coping de reenquadramento $(Z=-3.12$, $\mathrm{p} \leq .005)$ e do desempenhos físico $(\mathrm{Z}=-2.63$, $\mathrm{p} \leq .05)$ e emocional $(\mathrm{Z}=-2.62, \mathrm{p} \leq .05)$ em termos de qualidade de vida. Fumadores casados ou em união de fato relatam mais procura de coping de apoio espiritual. Quanto aos abstinentes, são também os casados, assim como os que vivem em união de fato, que melhor utilizam a estratégia de coping de reenquadramento e relatam melhor desempenho físico e emocional ao nível da qualidade de vida física e mental, respectivamente.

No que se refere às diferenças nas variáveis psicológicas em função de ter ou não doença respiratória crónica, observam-se diferenças entre abstinentes com e sem doença respiratória crónica ao nível da qualidade de vida física e mental e da morbilidade psicológica. Os primeiros percepcionam reduzida qualidade de vida e elevada morbilidade psicológica. Ao nível do coping, verificaram-se diferenças na avaliação passiva $(\mathrm{Z}=-2.41, \mathrm{p} \leq .05)$ que é mais utilizada por abstinentes com doença crónica.

\section{Preditores da qualidade de vida em fumadores e abstinentes}

Os resultados evidenciados na tabela 1 mostram que ter companheiro fumador revelase como preditor da qualidade de vida física dos fumadores ao explicar cerca de $3 \%$ da sua variância. No bloco II, ter companheiro fumador continua a revelar-se como preditor, bem como a morbilidade psicológica. Assim, se o parceiro não fumar, melhor tende a ser a qualidade de vida física do fumador e quanto mais grave a morbilidade psicológica, pior a sua qualidade de vida física. Por sua vez, a dependência da nicotina não prevê a qualidade de vida física. Porém, em conjunto, estas três variáveis explicam $21.6 \%$ da variância da qualidade de vida física dos fumadores $\left(\mathrm{F}_{(3.112)}=\right.$ $11,54, \mathrm{p} \leq .001)$.

Quanto à qualidade de vida mental dos fumadores, verificaram-se relações com a morbilidade psicológica e a avaliação passiva. Este modelo de regressão explica $31.2 \%$ da variância da qualidade de vida mental dos fumadores, mas individualmente, apenas a morbilidade psicológica revela-se como preditora de menor qualidade de vida mental.

Tabela 1 - Preditores da qualidade de vida nos fumadores $(\mathrm{N}=116)$.

\begin{tabular}{|c|c|c|c|c|c|c|c|c|}
\hline & \multicolumn{4}{|c|}{ Qualidade de Vida Física } & \multicolumn{4}{|c|}{ Qualidade de Vida Mental } \\
\hline & $\mathbf{R}^{2}$ & B & DP B & $\beta_{\text {std }}$ & $\mathbf{R}^{2}$ & B & DP B & $\beta_{\text {std }}$ \\
\hline $\begin{array}{l}\text { Bloco } 1 \\
\text { Hábito Tabágico do Companheiro }\end{array}$ & .04 & 3.39 & 1.41 & $.19^{*}$ & & - & - & - \\
\hline
\end{tabular}

R² Ajust: .03

Bloco 2

Hábito Tabágico do Companheiro

Dependência da Nicotina

Morbilidade Psicológica

Avaliação Passiva
.24

$\begin{array}{lrc}3.64 & 1.30 & .23^{*} \\ -.25 & .30 & -.10 \\ -.45 & .10 & -.42^{* *}\end{array}$

.33

$\begin{array}{ccc}- & - & - \\ - & - & - \\ -.67 & .11 & -.52^{* * *} \\ -.35 & .20 & -.14 \\ & & R^{2} \text { Ajust: . } 22\end{array}$

$\mathrm{p} \leq .05 ;{ }^{* * * *} \mathrm{p} \leq .001$ 
Relativamente aos abstinentes, como se pode observar na tabela 2 , no bloco I, a variável ter doença respiratória crónica explica $11.4 \%$ da variância da qualidade de vida física dos abstinentes, sendo que abstinentes sem doença respiratória crónica tendem a apresentar melhor qualidade de vida física. No bloco II, a variável ter doença respiratória crónica continua a revelar-se como preditora, assim como a morbilidade psicológica e o coping de procura de apoio espiritual. Os dados apresentados revelam que ter doença crónica, possuir elevados níveis de morbilidade psicológica e uma maior procura de apoio espiritual, no grupo dos abstinentes estão associados a pior qualidade de vida física, enquanto o coping de avaliação passiva não se revelou como preditor. Relativamente à qualidade de vida mental dos abstinentes, os resultados indicam que ser do sexo feminino, ter doença respiratória crónica e elevados níveis de morbilidade psicológica predizem pior qualidade de vida mental, explicando $29 \%$ da variância total.

Tabela 2 - Preditores da qualidade de vida nos abstinentes ( $N=117)$

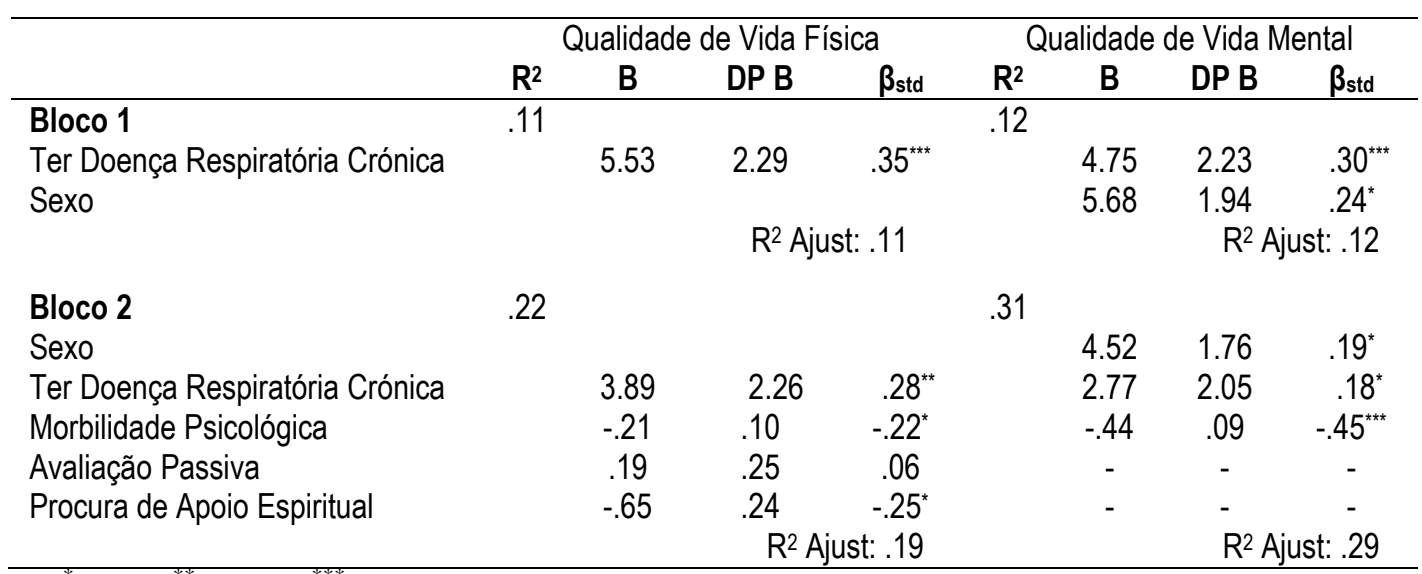

$\mathrm{p} \leq .05 ;{ }^{* *} \mathrm{p} \leq .005 ;{ }^{* * *} \mathrm{p} \leq .001$

\section{Discussão}

De acordo com os dados de investigações prévias, verificou-se que, em fumadores e abstinentes, os homens tendem a apresentar mais dependência nicotínica que as mulheres (Martinet \& Bohadana, 2003). Nos fumadores, os dados vão ao encontro da literatura existente. As mulheres procuram obter mais apoio social como estratégia de coping no processo de desabituação tabágica (Stewart, Thomas, \& Copeland, 2010). Quanto aos abstinentes, os homens apresentam menos morbilidade psicológica e melhor qualidade de vida mental (Dube et al., 2009) e nos abstinentes, as mulheres são mais propensas a desenvolver sintomas depressivos, pois, independentemente de terem história de comportamento tabágico, apresentam um risco duas vezes superior de terem sintomas depressivos (Angst et al., 2002).

Quanto à idade, abstinentes maiores de 40 anos, enquanto fumadores, possuíam mais dependência nicotínica que abstinentes de 40 ou menos anos, o que está de acordo a literatura, pois a longa duração de consumo de tabaco associa-se a níveis elevados de dependência nicotínica (Lubman, Yücel, \& Pantelis, 2004; Pratt et al., 2007).

No referente aos anos de escolaridade, em ambos os grupos, os níveis de dependência da nicotina tendem a ser mais elevados em indivíduos que possuem até 9 anos de escolaridade, o que está de acordo com a literatura. Os indivíduos com poucos anos de escolaridade tendem a apresentar baixo nível socioeconómico (NSE) e a este estatuto social estão associadas fontes de stress que aumentam a prática de comportamentos de risco como o tabagismo, o que resulta na forte dependência da nicotina (Kassel, Stroud \& Paronis, 2003). Os fumadores da amostra que possuem até 9 anos de escolaridade, tendem a procurar mais apoio espiritual como estratégia de coping. Estes dados estão de acordo com os resultados do estudo de Zavala, Maliski, Kwan, Fink e Litwin (2009), que verificaram que indivíduos com escolaridade inferior ao nível secundário 
relatam níveis mais elevados de espiritualidade. Por sua vez, os abstinentes com menos de 9 anos de instrução referem pior qualidade de vida física, o que faz sentido, tendo em consideração que nesta análise de diferenças não foi possível controlar a idade, nem a presença de doença crónica, o que pode explicar a reduzida qualidade de vida física dos abstinentes da nossa amostra. A investigação revela que após 20 a 30 anos de tabagismo, são visíveis problemas físicos graves (Machain et al., 2008) e o diagnóstico de uma doença grave e crónica associada ao tabagismo contribui para a decisão da cessação tabágica. No estudo de Trigo e Rocha (2002), a ocorrência de um enfarte agudo do miocárdio motivou a cessação tabágica dos pacientes.

Quanto ao estado civil, os resultados confirmam que fumadores e abstinentes casados ou em união de fato tendem a usufruir das estratégias de coping familiar de procura de apoio espiritual e reenquadramento, respectivamente, o que é congruente com a literatura que revela que o apoio social recebido promove a satisfação com a vida e o bem-estar e diminui a ocorrência de comportamentos de risco (Waldron \& Lye, 1989). A vinculação e envolvimento em atividades espirituais estimulam o sentido de comunidade, estando inversamente relacionadas com o comportamento de fumar (Turner-Musa \& Lipscomb, 2007). Além disso, o apoio do parceiro é um forte preditor da cessação tabágica bem sucedida (Park, Tudiver, Schultz \& Campbell, 2004).

No que toca aos abstinentes, são também os casados e os que vivem em união de fato que possuem melhor desempenho físico e emocional em termos de qualidade de vida. Segundo August e Sorkin (2010), o casamento tem um efeito protetor da saúde.

Foram ainda corroborados os dados da investigação indicativos de que abstinentes com doença respiratória crónica apresentam pior qualidade de vida, níveis mais elevados de morbilidade psicológica (Chapman, Perry \& Strine, 2005) e mais estratégias de coping passivas que os abstinentes sem doença respiratória crónica. Abstinentes com longa história de consumo de tabaco tendem a apresentar uma saúde física e emocional debilitada, podendo percecpionar apenas ténues melhorias na sua qualidade de vida física (Sarna et al., 2008). Assim, as suas expectativas de melhoria na qualidade de vida ficam reduzidas, levando-os a optar por estratégias de coping de evitamento (Friedman-Wheeler, Haaga, Gunthert, Ahrens \& McIntosh, 2008).

Relativamente aos preditores, constatamos que, no que concerne à qualidade de vida física, se o fumador convive com um parceiro que também fuma, a sua qualidade de vida tende a ser pior do que se convivesse com um parceiro não fumador. Todavia, se além de ter um parceiro fumador, o indivíduo tiver elevados níveis de dependência da nicotina e morbilidade psicológica, a sua qualidade de vida física tende a agravar-se. A investigação tem demonstrado que viver com um parceiro não fumador pode influenciar o fumador a adotar comportamentos de saúde (Homish \& Leonard, 2005), sendo que ter um companheiro não fumador ou abstinente está inclusivamente relacionado com uma probabilidade aumentada de sucesso na cessação tabágica (Monden et al., 2003). Por outro lado, se ambos forem fumadores, a cessação tabágica torna-se menos provável de ocorrer (Walsh et al., 2007). Quanto à dependência da nicotina e à morbilidade psicológica, verificamos que influenciam negativamente a qualidade de vida física dos fumadores, no sentido em que elevados níveis de dependência da nicotina e de morbilidade psicológica predizem pior qualidade de vida física nos fumadores. $\mathrm{O}$ consumo de nicotina relaciona-se com o desenvolvimento de doenças respiratórias, vários tipos de cancro, doenças cardiovasculares, entre outras (Doherty et al., 2009). No que se relacionada com a morbilidade psicológica, os resultados estão em conformidade com o que é referido na literatura, indicando que os fumadores com depressão major (Machain et al., 2008), stress psicológico (Manning, Catley, Harris, Mayo, \& Ahluwalia, 2005), ansiedade ou outra perturbação psicológica, tendem a ser mais dependentes da nicotina, consumindo em maiores quantidades e apresentam pior qualidade de vida física que os fumadores da população geral (Williams \& Ziedonis, 2004). A literatura evidencia também que, à medida que aumenta a duração do consumo e os níveis de dependência, são agravados os problemas de saúde mental, devido a problemas relacionados com ter uma doença crónica, como elevados gastos nos cuidados de saúde e o stress que uma pobre qualidade de vida física pode causar na família e no indivíduo (Machain et al., 2008). De fato, no referente à qualidade de vida mental, no nosso estudo, verificamos que 
fumadores com mais morbilidade psicológica e mais avaliação passiva, em termos de coping, apresentam pior qualidade de vida mental. Segundo Ismail, Sloggett e Stavola (2000), indivíduos deprimidos têm baixa autoestima, são pessimistas e tornam-se negligentes relativamente à sua saúde, adotando comportamentos de risco, como fumar. Segundo Spring et al. (2008), em fumadores deprimidos, o comportamento tabágico agrava $o$ afeto negativo. Outros fumadores relatam que usam o tabaco como meio para reduzir a ansiedade para atenuar as anomalias afetivas e neurobiológicas associadas ao seu problema psicológico (Feldner et al., 2009). Assim, a elevada prevalência do tabagismo na população com morbilidade psicológica leva a que esteja sob um risco aumentado de ter pior qualidade de vida (Perkins et al., 2010). Tal como já foi referido, no nosso estudo, a avaliação passiva, revelou-se como preditora da qualidade de vida mental dos fumadores, corroborando assim, o que é mencionado na literatura. Os fumadores com humor deprimido são menos ativos na utilização de estratégias de coping (Kinnunen, Doherty, Militello \& Garvey, 1996) e quando o fazem, tendem a adotar estratégias de evitamento, negação (avaliação passiva) e centradas na emoção, que, segundo Larsen (2000), são ineficazes para a regulação a longo prazo do humor. Relativamente aos abstinentes, concluímos que ter doença respiratória crónica, elevada morbilidade psicológica, bem como maior procura de apoio espiritual predizem pior qualidade de vida física. Os abstinentes apresentam maior tendência para serem portadores de doenças crónicas relacionadas com o tabagismo, que muitas das vezes são indicadas como um dos principais motivos da cessação tabágica (McClave, Dube, Strine, \& Mokdad, 2009). A literatura mostra que abstinentes referem menos morbilidade psicológica que os fumadores. Não obstante, ser portador de doença crónica tende a relacionar-se com morbilidade psicológica e pior qualidade de vida física (Dube et al., 2009).

Por seu turno, maior procura de apoio espiritual revela-se como preditora de reduzida qualidade de vida física nos abstinentes. Esta é uma estratégia de coping passivo, na medida em que é utilizada pelo indivíduo nos momentos em que não percepciona controlo sobre a situação de stress (Friedman-Wheeler et al., 2008). Assim, podemos interpretar que a procura de apoio espiritual é uma forma de lidar mais facilmente com a doença crónica ao facultar um significado e um propósito à vida do indivíduo face ao seu problema de saúde (Bekelman et al., 2009). Finalmente, os dados revelam que no grupo dos abstinentes, tal como é referido na literatura, ser do sexo feminino (Kendler et al., 1993), possuir doença respiratória crónica e elevada morbilidade psicológica predizem pior qualidade de vida mental (Wagena et al, 2004).

\section{Conclusão}

O presente estudo revelou a importância do coping familiar e da influência do parceiro no comportamento tabágico e na abstinência. Neste sentido, torna-se pertinente a construção de programas de intervenção no tabagismo que incluam os parceiros. Deve-se investir na promoção da autoeficácia em fumadores e abstinentes, reforçando a necessidade de sensibilização e formação de profissionais de saúde a este nível.

Investigações vindouras devem incidir na associação da dependência nicotínica com variáveis sociocognitivas implicadas no processo decisional de mudança de comportamento, estilo de vida e motivação para deixar de fumar, com o objetivo de melhorar e construir programas de intervenção mais eficazes no tabagismo.

\section{Referências}

Anda, R. F., Wiliamson, D. F., Escobedo, L. G., Mast, E. E., Giovino, G. A., Remington, P. L. (1990). Depression and the dinamics of smoking. A national perspective. Journal of the American Medical Association, 264(12), 1541-1545.

Angst, J., Gamma, A., Gastpar, M., Lépine, J. P., Mendlewicz, J., \& Tylee, A. (2002). Gender differences in depression: epidemiological findings from the European DEPRESS I and II studies. European Archives of Psychiatry Clinical Neuroscience, 252(5), 201-209.

August, K.J., \& Sorkin, D.H. (2010). Marital status and gender differences in managing a chronic illness: The function of healthrelated social control. Social Science \& Medicine, 71(10), 1831-1838. 
Bekelman, D. B., Rumsfeld, J. S., Havranek, E. P., Yamashita, T. E., Hutt, E., Gottlieb, S. H., et al. (2009). Symptom burden, depression, and spiritual well-being: A comparison of Heart failure and advanced cancer patients. Journal of Internal Medicine, 24(5), 592-598.

Benowitz, N. L. (1999). Nicotine addiction. Primary Care, 26(3), 611-631.

Benowitz, N. L. (2008). Clinical pharmacology of nicotine: implications for understanding, preventing, and treating tobacco addiction. Clinical Pharmacology and Therapeutics, 83(4), 531-541.

Bergen, A. W., \& Caporaso, N. (1999). Cigarrette smoking. Journal of the Nacional Cancer Institute,91(16), 1365-1375.

Chapman, D. P., Perry, G. S., \& Strine, T. W. (2005). The vital link between chronic disease and depressive disorders. Preventing Chronic Disease, 2(1), 1-10.

Clark, L., \& Watson, D. (1991). Tripartide model of anxiety and depression: psychometric evidence and taxonomic implications. Journal of Abnormal Psychology, 100(3), 316-336.

Direcção Geral de Saúde (DGS). (2009). Elementos estatísticos. Informação geral de saúde/2007. Lisboa: Divisão de Estatística de Saúde, Direcção Geral de Saúde. Recuperado em 20 de Setembro, 2010, de http://www.dgs.pt/

Doherty, J., Grabowski, J., Hoffman, C., Zelikoff, J. T. (2009). Early life insult from cigarette smoke may be predictive of chronic diseases later in life. Biomarkers, 14(S1), 97-101.

Dube, S. R., Caraballo, R. S., Dhingra, S. S., Pearson, W. S., McClave, A. K., Strine, T. W., et al. (2009). The relationship between smoking status and serious psychological distress: findings from the 2007 Behavioral Risk Factor Surveillance System. International Journal of Public Health, 54, 68-74.
Efstratiadis, S., Kennard, E. D., Kelsey, S. F., \& Michaels, A. D. (2008). Passive tobacco exposure may impair symptomatic improvement in patients with chronic angina undergoing enhanced external counterpulsation. BMC Cardiovascular Disorders, 8(23). doi: 10.1186/1471-22618-23

Ezquerra, E. A. (2003). Tabagismo e doença. Navarra, Espanha: Everest Editora.

Fagerström, K. O. (1978). Measuring degree of physical dependency to tobacco smoking with reference to individualization of treatment. Addictive Behaviours, 3, 235241.

Fagerström, K. O., \& Schneider, N. G. (1990). Measuring nicotine dependence: a reveiew of the Fagerström Tolerance Questionnaire. Journal of Behaviour Medicine,12, 159-181.

Feldner, M. T., Smith, R. C., Babson, K. A., Sachs-Ericsson, N., Schmidt, N. B., \& Zvolensky, M. J. (2009). Test of the role of nicotine dependence in the relation between posttraumatic stress disorder and panic spectrum problems. Journal of traumatic stress, 22(1), 36-44.

Ferreira, P. L., \& Santana, P. (2003). Perceção de estado de saúde e de qualidade de vida da população activa: Contributos para a definição de normas portuguesas. Revista Portuguesa de Saúde Pública, 21(2), 15-30.

Fisher, J., \& Corcoran, K. (2007). Measures for clinical practice and research. Couples, families and children ( $4^{\mathrm{a}}$ Ed.). New York: Oxford University Press.

Foulds, J., \& Ghodse, A. H. (1995). The role of nicotine in tobacco smoking: implications for tobacco control policy. Journal of the Royal Society of Health, 115(4), 225-230.

Friedman-Wheeler, D. G., Haaga, D. A., Gunthert, K. C., Ahrens, A. H., \& McIntosh, E. (2008). Depression, Neuroticism, and mood-regulation, expectancies for engagement and disengagement coping among cigarette smokers. Cognitive Therapy and Research, 32, 105-115. 
Gonzalez, A., Zvolensky, M. J., Vujanovic, A. A., Leyro, T. M., \& Marshall, E. C. (2009). Am evaluation of anxiety sensitivity, emotional dysregulation, and negative affectiveity among daily cigarette smokers: Relation to smoking motives and barriers to quitting. Journal of Psychiatric Research, $43,138-147$.

Graham, H. (1993) When life's a drag: women, smoking and disadvantage. London.

Hagman, B. T., Delnevo, C. D., Hrywna, M., \& Williams, J. M. (2007). Tobacco use among those with serious psychological distress: Results from the National Survey of Drug Use and Health, 2002. Addictive Behavior, 33(4), 582-592.

Holm, K. E., LaChance, H. R., Bowler, R. P., Make, B. J., \& Wamboldt, F. S. (2010). Family factors are associated with psychological distress and smoking status in chronic obstructive pulmonary disease. General hospital psychiatry, 32, 482-498.

Homish, G. G. \& Leonard, K. E. (2005). Spousal influence on smoking behaviors in a US community sample of newly married couples. Social Science \& Medicine, 61, 2557-2567.

Ismail, K., Sloggett, A., \& Stavola, B. (2000). Do common mental disorders increase cigarette smoking? Results from five waves of a population-based panel cohort study. American Journal of Epidemiology, 152(7), 651-657.

Kassel, J. D., Stroud, L. R., \& Paronis, C. A. (2003). Smoking, stress, and negative affect: correlation, causation, and context across stages of smoking. Psychological Bulletin, 129, 270-304.

Kendler, K. S., Neale, M. C., McLean, C. J., Heath, A. C., Eaves, L. J., \& Kessler, R. C. (1993). Smoking and major depression: a casual analysis. Archives of General Psychiatry, 50, 36-43.

Kershaw, T., Northouse, L., Kritpracha, C., Shafenacker, A., \& Mood, D. (2004). Coping strategies and quality of life in women with advanced breast cancer and their family caregivers. Psychology Health, 19, 149-156.
Kinnunen, T., Doherty, K., Militello, F.S., \& Garvey, A.J. (1996). Depression and smoking cessation: characteristics of depressed smokers and effects of nicotine replacement. Journal of Consulting \& Climical Psychology, 64, 791-798.

Larsen, R. J. (2000). Toward a science of mood regulation. Psychological Inquiry, 11, 129141.

Lovibond, P., \& Lovibond, S. (1995). The structure of negative emotional stats:comparison of the depression anxiety stress sc ales (DASS) with the Beck depression and anxiety inventories. Behaviour Research and Therapy, 33(3), 335-343.

Lubman, D.I., Yücel, M., \& Pantelis, C. (2004). Addiction, a condition of compulsive behavior? Neuroimaging and neuropsychological evidence of inhibitory dysregulation. Addiccion, 2004, 99(12), 1491-1502.

Machain, A. C., Vélez, N. A., García, F. J., Lugo, E. K., García, S. C., \& Mora, M. E. (2008). Relación entre el consumo de tabaco, salud mental y malestares físicos en hombres trabajadores de una empresa têxtil mexicana. Salud Mental, 31(4), 291-297.

Manning, B. K., Catley, D., Harris, K. J., Mayo, M. S. \& Ahluwalia, J. S. (2005). Stresss and quitting among African American smokers. Journal of Behavioral Medicine, 28(4), 325-333.

Martinet, Y., \& Bohadana, A. (2003). $O$ tabagismo. Da prevenção à abstinência. Lisboa: Climepsi Editores.

McClave, A., Dube, S., Strine, T., \& Mokdad, A. (2009). Associations between healthrelated quality of life and smoking status among a large sample of U.S. adults. Preventive Medicine, 48, 173-179.

McCubbin, H., Larsen, A., \& Olson, D. H. (1982). Family coping strategies (FCOPES). In H. I. McCubbin, H. Barnes, A. Larson, M. Muxen, \& M. Wilson (Eds.), Family inventories: Inventories used in a national survey of families across the family life cycle (pp. 101-119). St. Paul: University of Minnesota. 
McCubbin, H., Patterson, J., \& Sussman, M. (1983). Family stress process: the double ABCX model family adjustment and adaptation. Marriage and Family Review, 6, 7-38.

McDaniel, S., Campbell, T., Hepworth, J., \& Lorenz, A. (2005). Family - Oriented primary care ( $2^{\mathrm{a}} \mathrm{Ed}$.). New York: Springer.

Mendes, F., Relvas, A. P., Lourenço, M., Reccio, J. L., Pietralunga, S., Broyer, G., et al. (1999). Family relationships and primary prevention of drugs use and early adolescence. Valencia: IREFREA \& European Commissition.

Monden, W.S., de Graaf, N.D. \& Kraaykamp, G. (2003). How importante are parents and partners for smoking cessation in adulthood? An event history analysis. Preventive Medicine, 36(2), 223-273.

Pais-Ribeiro, J. L., Honrado, A., \& Leal, I. (2004). Contribuição para o estudo da adaptação portuguesa da escala de ansiedade, depressão e stress (EADS) de 21 itens de Lovibond e Lovibond. Psicologia, Saúde e Doença, 5(2), 229-239.

Park, E.,Tudiver, F., Schultz, J. K., \& Campbell, T. (2004). Does Enhancing Partner Support and interaction improve smoking cessation? A meta-analysis. Annals of Family Medicine, 2(2), 170-174.

Patton, G. C., Carlin, J. B., Coffey, C., Wolfe, R., Hibbert, M., \& Bowes, G. (1998). Depression, anxiety and smoking initiation: a prospective study over 3 years. American Journal of Public Health, 88(10), 15181522.

Pereira, M. G., \& Afonso, M. F. (2008a). Fagerström Test for Nicotine Dependence. Versão de Investigação. Universidade do Minho, Braga, Portugal.

Pereira, M. G., \& Afonso, M. F. (2008b). Questionário Sócio-Demográfico. Versão de Investigação. Universidade do Minho, Braga, Portugal.
Perkins, K. A., Karelitz, J. L., Giedgowd, G. E., Conklin, C. A., \& Sayette, M. A. (2010). Differences in negative mood-induced smoking reinforcement due to distress tolerance, anxiety sensitivity, and depression history. Psychopharmacology, $210,25-34$

Pratt, L. A., Day, A. N., \& Cohen, A. J. (2007). Caracteristics of adults with serious psychological distress as measured by the K6 scale: United States, 2001-04. Advance Data from Vital and Health Statistics, 30(382), 1-18.

Rüge, J., Ulbricht, S., Schummann, A., Rumpf, H.J., John, U., \& Meyer, C. (2008). Intention to quit smoking: Is the partners smoking status associated with the smoker's interntion to quit? International Journal of Behavioral Medicine, 15, 328-325.

Sarna, L., Bialous, S., Cooley, M., Jun, H., Feskanich, D. (2008). Impact of smoking cessation on health-related quality of life in women in the Nurses'Health Study. Quality of Life Research, 17, 1217-1227.

Schmitz, N., Kruse, J. \& Kugler, J. (2003). Disabilities, quality of life and mental disorders associated with smoking and nicotine dependence. American Journal of Psychiatry, 160(9), 1670-1676.

Siahpush, M., Spittal, M. M., \& Singh, G. K. (2007). Association of smoking cessation with financial stress and material wellbeing: Results from a prospective study of a population-based national survey. American Journal of Public Health, 97(12), 22812287.

Spring, B., Cook, J. W., Appelhans, B., Maloney, A., Richmond, M., Vaughn, J. et al. (2008). Nicotine effects on affective response in depression-prone smokers. Psychopharmacology, 196, 461-471.

Stewart, D. W., Thomas, J. L., \& Copeland, A. L. (2010). Percepcions of social support provided to smokers. Journal of Smoking Cessation, 5(1), 95-101.

Trigo, M., \& Rocha, E. (2002). Cessação de hábitos tabágicos em doentes coronários. Acta Médica Portuguesa, 5(5), 337-344. 
Tonstad, S. (2009). Cigarette smoking, smoking cessation and diabetes. Diabetes Research and Clinical Practice, 85, 4-13.

Turner-Musa, J., \& Lipscomb, L. S. (2007). Spirituality and social support on health behaviors of african american undergraduates. American Journal of American Behavior, 31(5), 495-501.

Wagena, E. J., Kant, I., Amelsvoort, L. G., Wouters, E. F., Schayck, C. P., \& Swaen, G. M. (2004). Risk of depression and anxiety in employees with chronic Bronchities:the modifying effect of cigarette smoking. Psychosomatic Medicine, 66, 729-734.

Waldron, I., \& Lye, D. (1989). Family roles and smoking. American Journal of Preventive Medicine, 5(3), 136-141.

Walsh, P. M., Carrillo, P., Flores, G., Masuet, C., Morchon, S., \& Ramon, J. M. (2007). Effects of partner smoking status and gender on long term abstinence rates of patients receiving smoking cessation treatment. Adictive Behaviors, 32(1), 128-136.

Ware, J. E., \& Sherbourne, C. D. (1992). The MOS 36-item short-form health survey (SF36). I. Conceptual framework and item selection. Medical Care, 30(6), 473-483.
Weinberger, A. H., Desai, R. A.,\& Mckee, S. A. (2010). Nicotine withdrawal in U.S. smokers with current mood, anxiety, alcohol use, and substance use disorders. Drug and Alcohol Dependence, 108, 7-12.

Williams, J. M., \& Ziedonis, D. (2004). Addressing tobacco among individuals with a mental illness or an addiction. Addictive Behaviors, 29, 1067-1083.

Wonnacott, S., Drasdo, A., Sanderson, E., \& Rowell, P. (1990). Presynaptic nicotinic receptors and the modulation of transmitter release. Ciba Foundation Symposium, 152, 87-101.

World Health Organization (WHO). (2002). The world health report: reducing risks and promoting healthy lifestyles. Genebra, Suiça.

Zavala, M. W., Maliski, S. L., Kwan, L., Fink, A., \& Litwin, M. S. (2009). Spirituality and quality of life in low-income men with metastatic prostate cancer. PsychoOncology, 18(7), 753-761.

Recebido em 01 de Novembro de 2011

Aceite em 23 de Maio de 2012

Publicado em 31 de Dezembro de 2012 